\title{
A Facile and Effective Method for Size Sorting of Large Flake Graphene Oxide
}

\author{
Ece Özçakır, Volkan Eskizeybek \\ Çanakkale Onsekiz Mart University, Department of Materials Science and Engineering \\ Department of Materials Science and Engineering, Çanakkale, Turkey \\ ozckrece@gmail.com; veskizeybek@comu.edu.tr
}

\begin{abstract}
The size of the building blocks fundamentally governs physical performances of the macro-scale graphene based structures since larger building blocks usually yields better mechanical and electrical properties. Density gradient centrifugation method has emerged as a versatile and scalable method for sorting colloidal 2D nanomaterials. This paper provides a facile and effective size sorting approach to graphene oxide (GO) flakes with large sizes up to $40 \mu \mathrm{m}$. The GO flakes were dispersed within distilled water. The centrifugation process parameters were calculated with respect to specific size ranges of GO flakes. Scanning electron microscopy utilized to prove the effectiveness of the separation process. Image processing analysis showed GO flakes with specific size ranges can be separate from aqueous suspension by controlling rotational speed and centrifugation time. The process was performed by using common benchtop centrifuges with low intensity centrifugal fields which requires low investment for a scalable process.
\end{abstract}

Keywords: Graphene oxide, centrifugation, size sorting, processing

\section{Introduction}

Graphene is a planar monolayer of carbon atoms that arranged into a two-dimensional honeycomb lattice with $\mathrm{sp}^{2}$ hybridized bonds [1]. The unique $\pi$-conjugation in graphene endows extraordinary mechanical, thermal and electrical properties which attract great scientific attention of many theoretical and experimental studies during recent years in various fields such as energy storage [2], electronic devices [3] and nanocomposites [4]. As a consequence, various graphene based structures in nano-, micro- and macro-scale have been synthesized and characterized, including 2D graphene papers [5] and $1 \mathrm{D}$ graphene fibers $[6,7]$.

The size of the building blocks fundamentally governs physical performances of the macro-scale graphene based structures since larger building blocks usually yields better mechanical and electrical properties up to three-fold increase [8, 9]. However, the difficulty of producing high quality graphene flakes with controlled size and chemical composition still remain as a challenge especially in scalable industrial processes. Chemical exfoliation of graphite with strong acid treatment, a technique introduced by Hummers and Brodie, one of the most preferred production method to generate graphene oxide (GO) flakes [10] which afford good dispersion within water and other commonly used solvents to form liquid crystal phases due to existence of epoxy and hydroxyl groups formed in the basal plane and carbonyl and carboxylic acid groups in the edge sites [11]. However, the oxidation and exfoliation processes of the graphite results diverse sizes of GO flakes. Therefore, the separation of the graphene based flakes with well-defined size is of importance for the accurate control of the macro-scale mechanical and electrical properties of the graphene based structures.

Centrifugal separation is a simple method used for size sorting of nano-scale materials distributed in liquid medium. It has been utilized generally as a post-synthesis size selection approach including GO flakes $[12,13]$. This work investigates how GO flakes distributed in aqueous medium can be sorted in definite size ranges before using to prepare macro-scale graphene based structures. First, centrifugation times for the specific size ranges are calculated based on Stokes' law to separate GO flakes from homogenously distributed aqueous medium. Next, GO/water suspensions are centrifuged by selecting appropriate angular velocity and centrifugation time. Scanning electron microscopy (SEM) is utilized to monitor centrifuged GO flakes and the size of GO flakes are calculated by image processing of SEM images and particle size analysis. 


\section{Theoretical Background}

In general, size sorting in centrifugal fields takes place when a difference in velocity between GO flakes of different sizes is generated [14]. Neglecting interactions between particles, viscosity increment of the solvent with addition of GO flakes and diffuse effects [14], the centrifugation time $(T, s)$ of a spherical particles in a liquid suspension is calculated by the following expression derived from Stokes's law;

$$
T=\frac{9 l \eta}{2 r^{2}\left(\rho_{p}-\rho_{s}\right) g}
$$

where $T$ (unit seconds) is time taken to precipitate suspended particles, $l$ is depth of solution $(m), r$ is radius of suspended particles $(\mathrm{m}), \eta$ viscosity of solution $(\mathrm{Pa} . \mathrm{s}), \rho_{p}$ density of suspended particles $\left(\mathrm{kg} \mathrm{m}^{-3}\right), \rho_{x}$ density of solution $\left(\mathrm{kg} \mathrm{m}^{-3}\right)$. Note that, Stokes' law is applied to spherical particles; however, GO flakes are in 2D morphology which are typically not spherical. Therefore, the particle radius in the regarding equation defines edge length of individual GO flakes assumed as squares.

As the rotor of centrifuge spins with a definite angular velocity, an apparent centrifugal force acts on the suspension and pushes radially outwards towards the side of the tube in the meantime. Schematic illustration of the centrifugation process is presented in Figure 1.

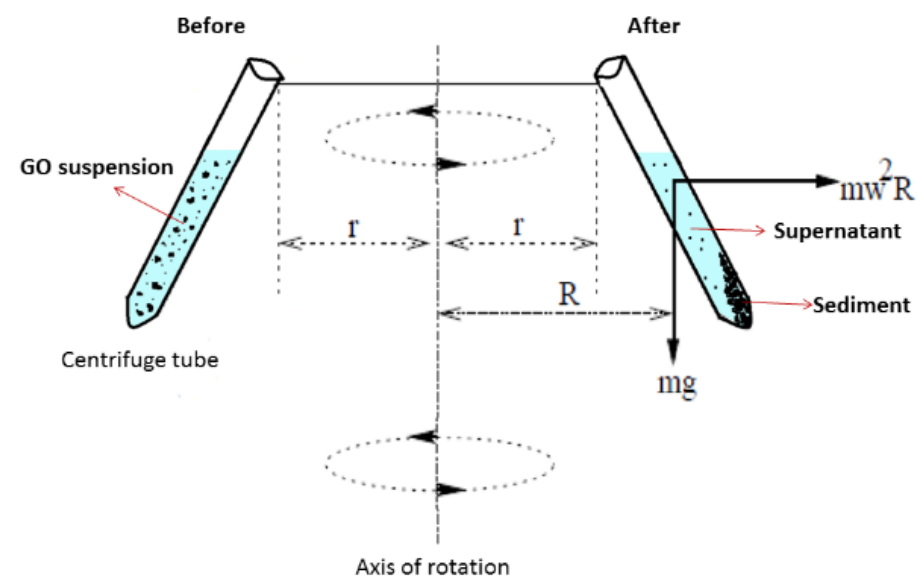

Fig. 1: Theory of centrifugation.

As seen in Figure 1, the force affected onto a particle in suspension during centrifugation depends both of the angular velocity $(\omega)$ and the radius of the centrifuge rotor $(R)$. Since the latter varies with different models of centrifuge, this makes it difficult to compare centrifuge times and speeds across different devices. This issue is resolved by quoting centrifugation in terms of relative centrifugal force $(R C F)$ which is defined as the ratio of the force acting on a particle by centrifugation to the force acting on the particle by gravity and expressed as follows;

$$
R C F=\frac{w^{2} R}{g}
$$

where $\omega$ angular velocity of centrifuge (radian $\left.s^{-1}\right), g$ gravitational acceleration $\left(m \cdot s^{-2}\right)$ and $R$ radius of the circular motion of the centrifuge $(m)$. However, Eqs. (2) is usually rearranged as;

$$
R C F=1.118 r\left(\frac{\mathrm{RPM}}{1000}\right)^{2}
$$


where RPM rotational speed (units revolutions per minute) and $r$ radius of the centrifuge rotor $(m)$ which is measured as $0.03 m$ in our case.

Thus, the centrifugation time can be expressed as;

$$
T=\frac{9 l \eta * R C F}{2 r^{2}\left(\rho_{p}-\rho_{s}\right) w^{2} R}
$$

Based on the Eqs. (4), the centrifugation time of the GO flakes with definite sizes can be calculated. The typical values used in Eqs (4) to calculate centrifugation time of the GO flakes with definite sizes are listed in Table 1.

Table 1. Typical values used calculate centrifugation time of the GO flakes with specific sizes.

\begin{tabular}{|c|l|l|}
\hline Symbol & \multicolumn{1}{|c|}{ Description } & \multicolumn{1}{|c|}{ Value } \\
\hline$l$ & Depth of solution $(\mathrm{m})$ & 0.08 \\
\hline$\eta$ & $\begin{array}{l}\text { Viscosity of water }\left(\text { at } 20{ }^{0} \mathrm{C}\right) \\
(\text { Pa.s })\end{array}$ & $1.002 \times 10^{-3}$ \\
\hline$r$ & Radius of GO $(\mathrm{m})$ & $5 \times 10^{-6}, 10 \times 10^{-6}, 15 \times 10^{-6}, 20 \times 10^{-6}, 25 \times 10^{-6}, 30 \times 10^{-6}, 35 \times 10^{-6}$ \\
\hline$\rho_{p}$ & Bulk density of $\mathrm{GO}\left(\mathrm{g} / \mathrm{cm}^{3)}\right.$ & $1.8\left(\right.$ at $\left.20^{\circ} \mathrm{C}\right)$ \\
\hline$\rho_{s}$ & Density of water $\left(\mathrm{g} / \mathrm{cm}^{3}\right)$ & $1.00\left(\right.$ at $20^{\circ} \mathrm{C}$ \\
\hline$R$ & Radius of rotation $(\mathrm{m})$ & 0.086 \\
\hline$w$ & Angular velocity $\left(\mathrm{rad} . \mathrm{s}^{-1}\right)$ & $\begin{array}{l}6000 \mathrm{RPM}\left(628.319 \mathrm{rad} . \mathrm{s}^{-1}\right) 5000 \mathrm{RPM}\left(523.599 \mathrm{rad} . \mathrm{s}^{-1}\right) 4000 \\
\mathrm{RPM}\left(418.879 \mathrm{rad} . \mathrm{s}^{-1}\right) 3000 \mathrm{RPM}\left(314.159 \mathrm{rad} . \mathrm{s}^{-1}\right) 2000 \mathrm{RPM} \\
\left(209.440 \mathrm{rad} . \mathrm{s}^{-1}\right)\end{array}$ \\
\hline
\end{tabular}

\section{Experimental Section}

\subsection{Separation of GO Flakes with Centrifugation Process}

GO flakes were purchased from Graphene Supermarket, USA (single layer nanoplatelets content >97\%) with flake sizes up to $40 \mu \mathrm{m}$. First $10 \mathrm{mg}$ of GO flakes were dispersed in $10 \mathrm{~mL}$ distilled water effectively by aid of sonication and magnetic stirring, respectively. Following, centrifugation process was utilized to separate GO flakes with specific sizes. Hettich EBA 20 centrifuge with a rotor radius $86 \mathrm{~mm}$ and a maximum rotational speed of $6000 \mathrm{rpm}$ was used during centrifugation process. The centrifugation times according to Eqs. (4) for fixed GO flake size ranges and rotational speeds are calculated and listed in Table 2. Since the regarding centrifuge allows to set centrifugation times as minutes, all of the calculated centrifugation times were represented in minutes. Note that, it takes thirty seconds for the centrifuge to accelerate and slow down and this time is not included into the calculated separation times.

Table 2. The calculated centrifugation times of the GO flakes with specific size ranges for the given rotational speeds.

\begin{tabular}{|c|r|r|r|r|r|r|}
\hline $\begin{array}{c}\text { Rotational } \\
\text { speeds }\end{array}$ & \multicolumn{7}{|c|}{ GO flake size ranges } \\
\hline & $\mathbf{0 - 5} \boldsymbol{\mu m}$ & $\mathbf{5 - 1 0 \mu \mathrm { m }}$ & $\mathbf{1 0 - 1 5} \boldsymbol{\mu m}$ & $\mathbf{1 5 - 2 0 \mu \mathrm { m }}$ & $\mathbf{2 0 - 2 5} \boldsymbol{\mu m}$ & $\mathbf{2 5 - 3 0 \mu \mathrm { m }}$ \\
\hline $\mathbf{6 0 0 0} \mathbf{~ r p m}$ & $6^{\prime}$ & $2^{\prime}$ & $1^{\prime}$ & & & \\
\hline $\mathbf{5 0 0 0} \mathbf{~ r p m}$ & $7^{\prime}$ & $2^{\prime}$ & $1^{\prime}$ & & & \\
\hline $\mathbf{4 0 0 0} \mathbf{~ r p m}$ & $9^{\prime}$ & $3^{\prime}$ & $1^{\prime}$ & & & \\
\hline $\mathbf{3 0 0 0} \mathbf{~ r p m}$ & $12^{\prime}$ & $3^{\prime}$ & $2^{\prime}$ & $1^{\prime}$ & & \\
\hline $\mathbf{2 0 0 0} \mathbf{~ r p m}$ & $17^{\prime}$ & $5^{\prime}$ & $2^{\prime}$ & $2^{\prime}$ & $1^{\prime}$ & \\
\hline $\mathbf{1 0 0 0} \mathbf{~ r p m}$ & $34^{\prime}$ & $9^{\prime}$ & $4^{\prime}$ & $3^{\prime}$ & $2^{\prime}$ & $1^{\prime}$ \\
\hline
\end{tabular}


The GO flakes were separated using centrifugation at different rotational speeds and centrifugation times according to Table 2 for specific flake sizes, and the supernatant solution was then decanted. The sediment was redispersed in $10 \mathrm{~mL}$ distilled water, and the centrifugation and decanting steps were repeated to prepare size-sorted GO flakes. The selected centrifugation process parameters for the flake size ranges as 0-5, 5-10 and 10-15 $\mu \mathrm{m}$ are $6000 \mathrm{rpm} / 6 \mathrm{~min}, 5000 \mathrm{rpm} / 2 \mathrm{~min}$ and $4000 \mathrm{rpm} / 1 \mathrm{~min}$, respectively. The typical separation process of GO flakes with specific size ranges is schematically illustrated in Figure 2. After separation, the supernatant solution was dried in silicon mould at $60^{\circ} \mathrm{C}$ for $4 \mathrm{~h}$ under vacuum conditions to obtain a powder for characterization. Since, the goal of the centrifugation process to separate at least half of the GO flakes with different flake size ranges, some process steps were repeated as shown in Figure 2.

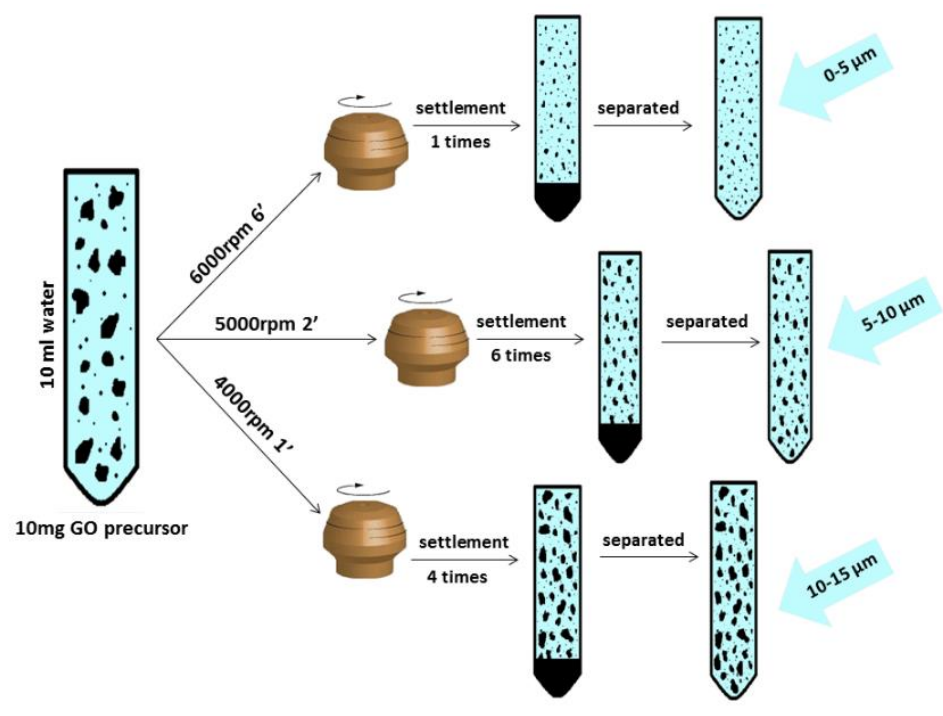

Fig. 2: Schematic representation of the centrifugation process utilized to separate GO flakes with specific sizes.

\subsection{Characterization}

The resulting dried GO flakes were monitored using scanning electron microscopy (JEOL SEM-7100-EDX). At least ten SEM images were recorded from different areas of the deposited samples on Si wafer, and image processing was applied to measure GO flake areas. Assuming GO flakes are square, the GO flake sizes were calculated from at least $400 \mathrm{GO}$ flakes. X-ray photoelectron spectroscopy (XPS) measurements were performed with a Thermo Scientific K-Alpha spectrometer using a monochromic Al Ka source at $1000 \mathrm{eV}$. Perkin Elmer 1725 FT-IR spectrometer with ATR unit was used during whole experiments with scanning range $550-4000 \mathrm{~cm}^{-1}$ at a resolution of $4 \mathrm{~cm}^{-1}$.

\section{Results and Discussions}

The size distribution of purchased GO flakes is represented in Figure 1a. According to the inset, the flake sizes of the GO precursor are mostly in the range of 5 to $15 \mu \mathrm{m}$. Therefore, we aimed to separate flakes smaller than $15 \mu \mathrm{m}$ by choosing appropriate centrifugation process parameters to separate half of the flakes from the water suspension. The SEM image of the GO precursor is shown in Figure 1b. The size distribution graph of the GO precursor was calculated by measuring more than 800 particles from different SEM images. Fig. 2b shows the FTIR spectra of GO precursor. The peaks at at $17200 \mathrm{~cm}^{-1}$ $\left(\mathrm{C}=\mathrm{O}\right.$ stretching vibrations), at $1616 \mathrm{~cm}^{-1}$ (skeletal vibrations $\left.\mathrm{C}-\mathrm{C}\right)$, at $1348 \mathrm{~cm}^{-1}$ (O-H stretching vibrations), at $1165 \mathrm{~cm}^{-1}$ (C-O-C stretching vibrations), at $1034 \mathrm{~cm}^{-1}$ (C-O stretching vibrations) are characteristic for the GO [15]. XPS survey spectrum (Figure 1d) shows the calculated value of $\mathrm{C} 1 \mathrm{~s} / \mathrm{O} 1 \mathrm{~s}$ (atomic ratios) was 2.08. Curve fitting of the $\mathrm{C} 1 \mathrm{~s}$ was performed using a Gaussian-Lorentzian peak shape. The peaks of $\mathrm{C} 1 \mathrm{~s}$ spectra are assigned to four components that correspond to carbon atoms in different functional groups: the nonoxygenated ring $\mathrm{C}(\mathrm{C}-\mathrm{C})$, the $\mathrm{C}$ in $\mathrm{C}-\mathrm{O}$ bonds $(\mathrm{C}-\mathrm{OH})$, the carbonyl $\mathrm{C}(\mathrm{C}=\mathrm{O})$, and the carboxylate carbon $(\mathrm{O}=\mathrm{C}-\mathrm{OH})[16]$.

Figure $4 \mathrm{a}$ shows the GO flakes in the test tube before centrifugation process. As indicated in Figure $4 \mathrm{~b}$, c, after centrifugation in the 5000 and $4000 \mathrm{rpm}$ the GO concentration in water suspension decreases since the light brown colour of the suspension appeared as lighter brown supernatants in colour. 

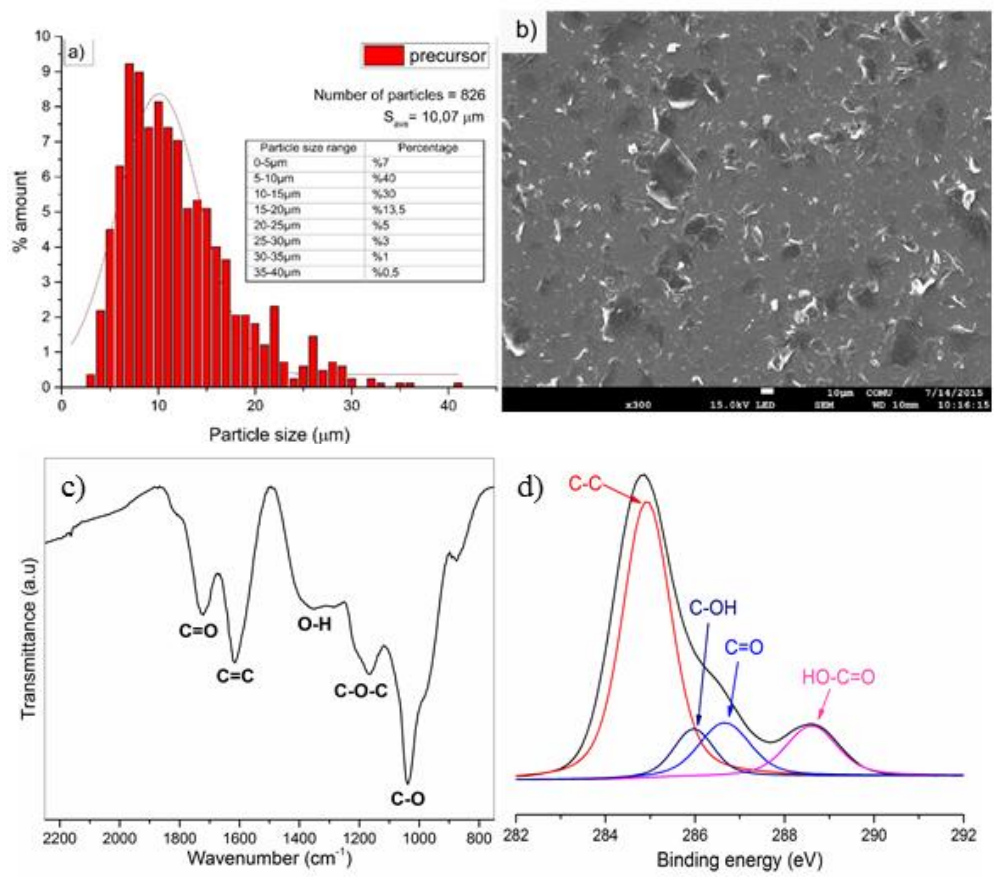

Fig. 3: The characteristic structural and morphological properties of GO precursor used in experiments, a) size distribution of GO flakes based on image processing analysis, b) A typical SEM image of GO precursor (scale bar $10 \mu \mathrm{m}$ ), c) FTIR spectrum the GO flakes, d) XPS spectrum of GO flakes.

(a)

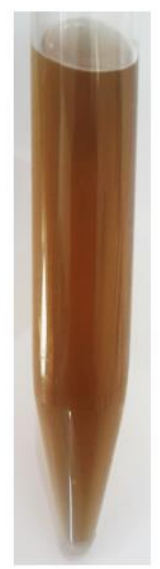

(b)

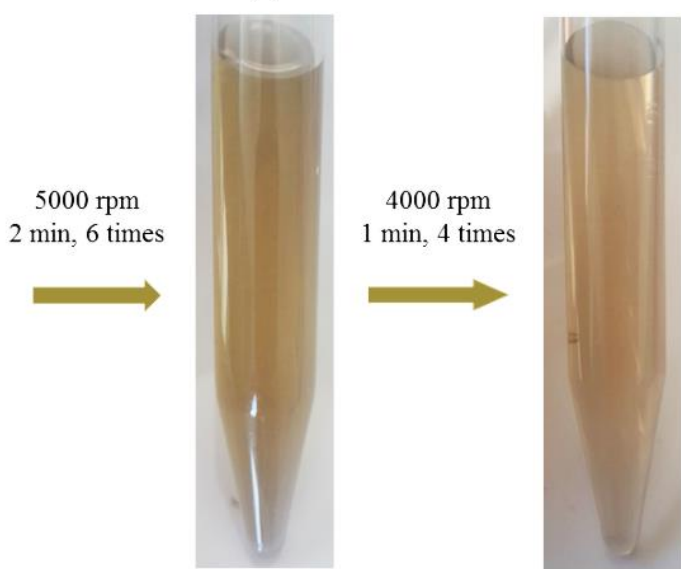

(c)

Fig. 4: Selective centrifugation of GO flakes in test tubes (a) before and (b,c) after centrifugation, in the (b) $5000 \mathrm{rpm}$ (c) $4000 \mathrm{rpm}$.

The rational speed and the centrifugation time are crucial parameters for size sorting of GO flakes. In particular, by increasing the rational speed and reducing the centrifugation time, the amount of sediment increases: by centrifugation either at $6000 \mathrm{rpm}$ for $6 \mathrm{~min}$, at $5000 \mathrm{rpm}$ for $2 \mathrm{~min}$, and at $4000 \mathrm{rpm}$ for $1 \mathrm{~min}$, we obtain, respectively, 6.15, 8.74 and $11.97 \mu \mathrm{m}$ of the average GO flake sizes as shown in Figure 5. In actuality, GO flakes smaller than $5 \mu \mathrm{m}$ were observed in all SEM images taken after second and third step of centrifugation process. This is attributed to brake of large GO flakes during preparing GO sediments for next separation process. It is known that bath-sonication mixing of GO flakes results decreasing sizes and consequently decreases the average flake size of the sediment. Therefore, we applied bath-sonication to disperse GO sediment in water for 5 min to minimize damage and following mixed with vortex shaker for 2 min.

After the separation of GO flakes with sizes smaller than $15 \mu \mathrm{m}$, the average size of GO flake sediment at the bottom of the tube is measured as $\sim 17 \mu \mathrm{m}$ as shown in Figure 6. This proves the effectiveness of selective centrifugation for GO flake size sorting, while requiring low centrifugal fields, achievable with ordinary benchtop centrifuges. It must be mentioned 
that particle size analysis of GO flakes was also performed for all cases to compare results obtained by image processing analysis; however, higher average flake sizes more than $20 \%$ of the regarding image processing results were obtained which was attributed to low concentration of GO flakes in water suspension.
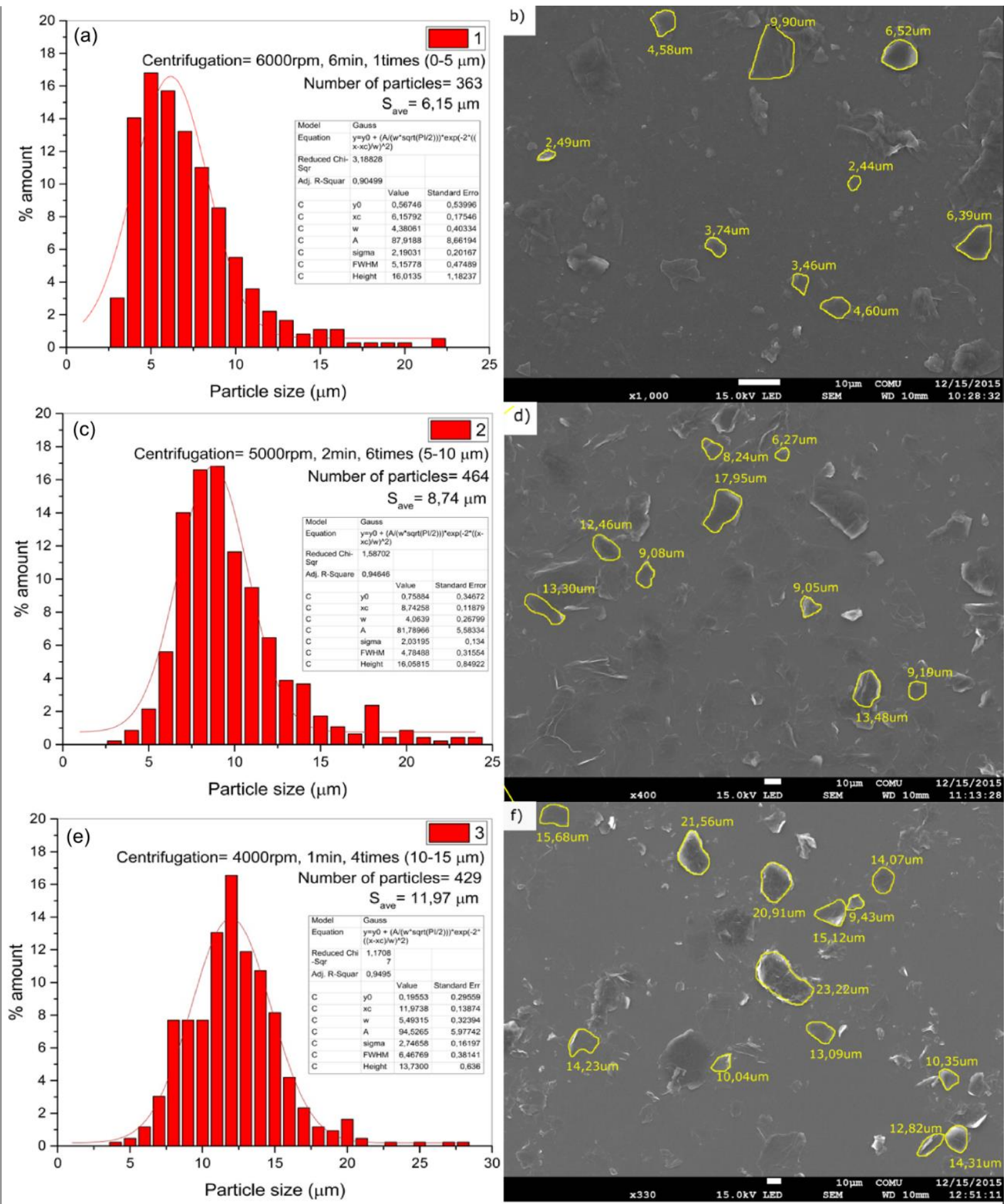

Fig. 5: The flake size distribution of separated GO supernatants a) $6000 \mathrm{rpm} 6 \mathrm{~min}$, c) $5000 \mathrm{rpm} 2 \mathrm{~min}$, e) $4000 \mathrm{rpm} 1 \mathrm{~min}$, and corresponding SEM images selected from image processing of the flakes (scale bar $10 \mu \mathrm{m}$ ), b) $6000 \mathrm{rpm} 6 \mathrm{~min}, \mathrm{~d}) 5000 \mathrm{rpm} 2 \mathrm{~min}, \mathrm{f}$ ) $4000 \mathrm{rpm} 1 \mathrm{~min}$. 

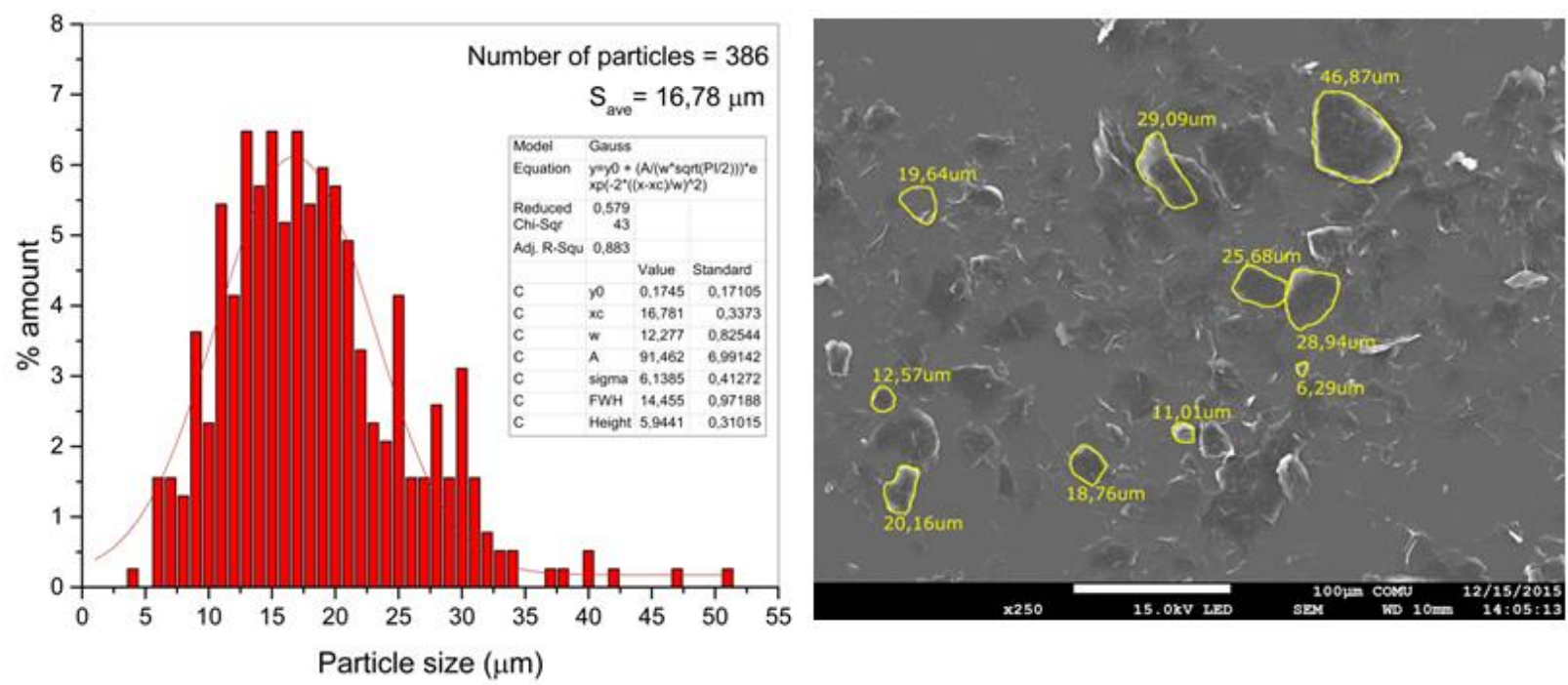

Fig. 6: The flake size distribution and corresponding SEM images selected from image processing of the flakes of GO sediment.

\section{Conclusion}

In this paper, it has been demonstrated the viability of centrifugation process for size selection of GO flakes with large sizes by in distilled water medium without the use of chemical additives or other solutes. The centrifugation process parameters rotational speed and centrifugation time were calculated correspond to specific GO flake sizes. The GO flakes with sizes up to $15 \mu \mathrm{m}$ were separated into three groups with average sizes 6,8 and $12 \mu \mathrm{m}$. In addition the average flake size of GO sediment was measured as $17 \mu \mathrm{m}$. The total calculated centrifugation time $9 \mathrm{~min}$ for three stepped separation process without repeat and mixing times; moreover, it takes approximately $90 \mathrm{~min}$ half of the matter for a single tube including 10 $\mathrm{mg}$ GO flakes. The proposed process to sort of GO flakes with specific size ranges effectively was implemented in common benchtop centrifuges with low intensity centrifugal fields which requires low investment for a scalable process.

\section{Acknowledgements}

We acknowledge financial supports from The Scientific and Technological Research Council of Turkey (TUBITAK) under grant no. MAG-214M650. We thank Çanakkale Onsekiz Mart University Science and Technology Application and Research Centre for support with SEM measurements.

\section{References}

[1] A. Esmaeili and M. H. Entezari, "Facile and fast synthesis of graphene oxide nanosheets via bath ultrasonic irradiation," Journal of Colloid and Interface Science, vol. 432, pp. 19-25, 2014.

[2] X. Tong, H. Wang, G. Wang, L. J. Wan, Z. Y. Ren, J. T. Bai, et al., "Controllable synthesis of graphene sheets with different numbers of layers and effect of the number of graphene layers on the specific capacity of anode material in lithium-ion batteries," Journal of Solid State Chemistry, vol. 184, pp. 982-989, 2011.

[3] X. M. Li, T. S. Zhao, Q. Chen, P. X. Li, K. L. Wang, M. L. Zhong, et al., "Flexible all solid-state supercapacitors based on chemical vapor deposition derived graphene fibers," Physical Chemistry Chemical Physics, vol. 15, pp. 1775217757, 2013.

[4] Q. Q. Zhuo, Y. Y. Ma, J. Gao, P. P. Zhang, Y. J. Xia, Y. M. Tian, et al., "Facile Synthesis of Graphene/Metal Nanoparticle Composites via Self-Catalysis Reduction at Room Temperature," Inorganic Chemistry, vol. 52, pp. 31413147, 2013.

[5] H. Chen, M. B. Muller, K. J. Gilmore, G. G. Wallace, and D. Li, "Mechanically strong, electrically conductive, and biocompatible graphene paper," Advanced Materials, vol. 20, pp. 3557-+, 2008.

[6] Z. Xu and C. Gao, "Graphene chiral liquid crystals and macroscopic assembled fibres," Nature Communications, vol. $2,2011$.

[7] H. H. Cheng, Z. L. Dong, C. G. Hu, Y. Zhao, Y. Hu, L. T. Qu, et al., "Textile electrodes woven by carbon nanotubegraphene hybrid fibers for flexible electrochemical capacitors," Nanoscale, vol. 5, pp. 3428-3434, 2013. 
[8] H. G. Chae and S. Kumar, "Materials science - Making strong fibers," Science, vol. 319, pp. 908-909, 2008.

[9] C. S. Xiang, C. C. Young, X. Wang, Z. Yan, C. C. Hwang, G. Cerioti, et al., "Large Flake Graphene Oxide Fibers with Unconventional 100\% Knot Efficiency and Highly Aligned Small Flake Graphene Oxide Fibers," Advanced Materials, vol. 25, pp. 4592-4597, 2013.

[10] M. J. Allen, V. C. Tung, and R. B. Kaner, "Honeycomb Carbon: A Review of Graphene," Chemical Reviews, vol. 110, pp. 132-145, 2010.

[11] S. H. Aboutalebi, M. M. Gudarzi, Q. B. Zheng, and J. K. Kim, "Spontaneous Formation of Liquid Crystals in Ultralarge Graphene Oxide Dispersions," Advanced Functional Materials, vol. 21, pp. 2978-2988, 2011.

[12] F. Bonaccorso, M. Zerbetto, A. C. Ferrari, and V. Amendola, "Sorting Nanoparticles by Centrifugal Fields in Clean Media," Journal of Physical Chemistry C, vol. 117, pp. 13217-13229, 2013.

[13] J. T. Han, J. I. Jang, S. H. Kim, S. Y. Jeong, H. J. Jeong, and G. W. Lee, "Size sorting of chemically modified graphene nanoplatelets," Carbon Letters, vol. 14, pp. 89-93, 2013.

[14] J. Bandrowski, H. Merta, and J. Ziolo, "Analysis of the Methods of Calculation of Continuous Sedimentation Area, Based on the Sedimentation Test of the Suspension .1. Theory of the Process," Inzynieria Chemiczna I Procesowa, vol. 14, pp. 555-568, 1993.

[15] D. Konios, M. M. Stylianakis, E. Stratakis, and E. Kymakis, "Dispersion behaviour of graphene oxide and reduced graphene oxide," Journal of Colloid and Interface Science, vol. 430, pp. 108-112, 2014.

[16] C. A. Tao, J. F. Wang, S. Q. Qin, Y. A. Lv, Y. Long, H. Zhu, et al., "Fabrication of pH-sensitive graphene oxide-drug supramolecular hydrogels as controlled release systems," Journal of Materials Chemistry, vol. 22, pp. 24856-24861, 2012. 